\title{
RECIST Comments
}

National Cancer Institute

\section{Source}

National Cancer Institute. RECIST Comments. NCI Thesaurus. Code C159894.

Any comments pertaining to the RECIST evaluation. 